\title{
Molecular Characterization of Mycobiota and Aspergillus Species from Eupolyphaga sinensis Walker Based on High- Throughput Sequencing of ITS1 and CaM
}

\author{
Ling Bai $\mathbb{D},{ }^{1,2}$ Liu He $\mathbb{D},{ }^{2}$ Penghao Yu, ${ }^{2}$ Jiaoyang Luo, ${ }^{2}$ Meihua Yang, ${ }^{2}$ Xiangren $A,{ }^{3}$ \\ and Xiaoxing Wei ${ }^{1,3}$ \\ ${ }^{1}$ College of Eco-Environmental Engineering, Qinghai University, Xining, Qinghai 810016, China \\ ${ }^{2}$ Institute of Medicinal Plant Development, Chinese Academy of Medical Sciences \& Peking Union Medical College, \\ Beijing 100193, China \\ ${ }^{3}$ Department of Clinical Laboratory Medicine, People's Hospital of Qinghai, Xining, Qinghai 810007, China
}

Correspondence should be addressed to Liu He; lhe@implad.ac.cn and Xiaoxing Wei; flemingo@126.com

Received 3 January 2020; Revised 28 February 2020; Accepted 9 March 2020; Published 7 May 2020

Academic Editor: Andrea Galimberti

Copyright $\odot 2020$ Ling Bai et al. This is an open access article distributed under the Creative Commons Attribution License, which permits unrestricted use, distribution, and reproduction in any medium, provided the original work is properly cited.

Eupolyphaga sinensis Walker is a valuable traditional Chinese animal medicine first recorded in Shennong Bencao. Previous research has shown that E. sinensis is easily contaminated by aflatoxins (AFs), which are highly toxic mycotoxins, during harvest, storage, and transport, thereby posing a considerable threat to consumer health. Most often, these AFs are produced by Aspergillus species. In this study, we contrast the traditional culture-based dilution plating method to the high-throughput sequencing (HTS) technology for fungal identification in TCM E. sinensis. Both of the methods used internal transcribed spacer 1 (ITS1) and calmodulin (CaM) sequencing for fungal molecular identification. The new CaM primer we designed in the study is suitable for MiSeq PE300 sequencing used for identification of Aspergillus species in community DNA samples. More fungal species were found in the E. sinensis samples based on HTS than those found using the culture-based dilution plating method. Overall, combining the sequencing power of ITS1 and $\mathrm{CaM}$ is an effective method for the detection and monitoring of potential toxigenic Aspergillus species in E. sinensis. In conclusion, HTS can be used to obtain a large amount of sequencing data about fungi contaminating animal medicine, allowing earlier detection of potential toxigenic fungi and ensuring the efficient production and safety of E. sinensis.

\section{Introduction}

The dried body of the female ground beetle Eupolyphaga sinensis Walker is a valuable traditional Chinese animal medicine first recorded in Shennong Bencao and has been used as a traditional Chinese medicine (TCM) for hundreds of years. The insect is collected, scalded to death by boiling water, and dried either in the sun or by baking. Due to its high protein content [1] and unique taste, it is also regarded as a high-class dish by some restaurants and customers. Therefore, with the demand increasing for E. sinensis, it has a substantial market potential in China as medicine and food. Currently, E. sinensis is raised through artificial breeding.

Because artificially cultivated E. sinensis is usually fed organic humus, such as wheat bran and decaying leaves, the feed remaining in the intestinal tract of the ground beetle is highly susceptible to mould and fungus contamination during the process of harvesting, processing, transporting, and storing the insect. According to market research, $E$. sinensis can easily be contaminated by aflatoxins [2-4], which affect the safety of the medicine and threaten the health of consumers [5, 6]. Aflatoxin is produced mainly by Aspergillus fungi [7]. The early detection of Aspergillus contaminants in E. sinensis can prevent mycotoxins from entering the consumer market. The rapid discovery of $A s-$ pergillus fungi is of great significance for ensuring the efficient production and safety of TCM.

The identification of Aspergillus sp. is based on morphology and molecular species recognition. DNA barcoding has become increasingly important for molecular 
identification of fungi and fungal ecological research. The nuclear rDNA internal transcribed spacer (ITS) region is the official DNA barcode for exploring fungal diversity and community in environmental samples because the ITS region can be readily amplified from most DNA samples with universal primers [8] and has the highest probability of identifying the broadest range of fungi [9]. However, ITS sequences lack sufficient variation to distinguish some Aspergillus clades [10]. Samson et al. recommend calmodulin (CaM) as a secondary identification marker for Aspergillus sp. [11]. So far, there has been limited research on CaM use for Aspergillus species identification in community DNA samples.

With the development of high-throughput sequencing (HTS) techniques, the number of large-scale studies of fungi occurring in association with plants [12, 13], animals [14], and human beings [15] has increased. Here, we designed a new primer CaMr1 paired with CMD5 [16] to acquire a CaM amplicon length of no more than $300 \mathrm{bp}$. The amplicon could then be used for MiSeq PE300 analysis of the Aspergillus species. We extracted fungal community DNA occurring throughout TCM E. sinensis samples and used HTS technology based on ITS1 and CaM amplicons to examine the fungal microbiome and Aspergillus sp. We also used culture-based dilution plating method to isolate fungi from E. sinensis. Compared with the traditional method, the HTS results for the two markers show that combining ITS1 and CaM for identifying fungi in TCM is very efficient for the discovery of potential toxigenic Aspergillus species.

\section{Materials and Methods}

2.1. Sample Preparation. E. sinensiswas purchased from Cao County (Shandong, China) and authenticated by Prof. Yulin Lin (Institute of Medicinal Plant Development, CAMS). Three samples of E. sinensis (ES1, ES2, and ES3) were used in both culture-based dilution plating method and HTS.

2.2. Culture-Based Dilution Plating Method. Each of the three E. sinensis samples $(4 \mathrm{~g})$ was crushed in a flask containing $40 \mathrm{~mL}$ of sterile water and 20 glass beads; the flask was shaken at $120 \mathrm{r} \cdot \mathrm{min}^{-1}$ for $5 \mathrm{~min}$ and allowed to stand for $5 \mathrm{~min}$. The suspension was diluted to $10^{-1} \sim 10^{-6}$, and $1 \mathrm{~mL}$ of each sample was applied to malt extract agar (MEA) medium containing $100 \mathrm{ppm}$ chloramphenicol, with three repeats for each dilute concentration. The plates were cultured at $25^{\circ} \mathrm{C}$, and colonies were counted and separated into single spores after seven days. The hyphae were collected and ground with liquid nitrogen in a mortar. The DNA was extracted with the M5 Fungal Genomic DNA Kit (Mei5 Biotech, China), and PCR was conducted using ITS primers (ITS1: TCCGTAGGTGAACCTGCGG and ITS4: TCCTCCGCTTATTGATATGC) [8] and CaM primers (CMD5: CCGAGTACAAGGARGCCTTC and CMD6: CCGATRGAGGTCATRACGTGG) [16], respectively. The PCR products were sequenced using Sanger sequencing.

\subsection{HTS Method}

2.3.1. DNA Extraction. The three E. sinensis samples, each weighing $40 \mathrm{~g}$, were ground with liquid nitrogen in a mortar. The total community DNA was extracted from $4 \mathrm{~g}$ of the resulting powder with the M5 Fungal Genomic DNA Kit (Mei5, China). The DNA concentration was measured with NanoDrop 2000 (Thermo Scientific, USA) and was $\geq 20 \mathrm{ng} \cdot \mu \mathrm{L}^{-1}$. The $\mathrm{OD}_{260} / \mathrm{OD}_{280}$ was between 1.8 and 2.0. The extracted DNA was stored at $-20^{\circ} \mathrm{C}$ for subsequent analyses.

2.3.2. ITS1 and CaM Primer Amplification. The DNA extracted from each sample was used as template DNA at $10 \mathrm{ng}$. Because the entire ITS region ranges between 450 and $700 \mathrm{bp}$ which is too long for HTS, a partial sequence was used. The primers ITS1-F (5'-CTTGGTCATTTAGAGGAAGTAA- $\left.3^{\prime}\right)$ [17] and ITS2 (5'-GCTGCGTTCTTCATCGATGC-3') $[8,18]$ were used for amplifying the ITS1 region. ITS1-F and ITS2 have been used to survey the diversity and composition of the fungal communities from environmental sample frequently during the last 10 years [19-21]. The primers CMD5 and CaMr1 (5'CCTTRGTRG TGA TYT GRC CT-3' ${ }^{\prime}$, this study) were used for amplifying a segment of CaM. PCRs were performed with Trans Start Fast Pfu DNA Polymerase (TransGen, China). The reaction system contained $4 \mu \mathrm{L} 5 \times$ Fast Pfu Buffer, $2 \mu \mathrm{L}$ dNTP $(2.5 \mathrm{mM}), 0.8 \mu \mathrm{L}$ each of the forward and reverse primers, $10 \mathrm{ng}$ of template DNA, $0.4 \mu \mathrm{L}$ Fast Pfu Polymerase, and ultrapure water to a final volume of $20 \mu \mathrm{L}$. PCR amplification was performed under the following conditions: $95^{\circ} \mathrm{C}$ for $5 \mathrm{~min} ; 95^{\circ} \mathrm{C}$ for $30 \mathrm{~s}, 55^{\circ} \mathrm{C}$ for $30 \mathrm{~s}$, and 27 cycles of $72^{\circ} \mathrm{C}$ for $45 \mathrm{~s}$; and $72^{\circ} \mathrm{C}$ for $10 \mathrm{~min}$. All samples were used in the experiment, and each sample was amplified three times. The PCR products of the same samples were mixed and detected by $2 \%$ agarose gel electrophoresis, and the PCR was recovered with the Axy Prep DNA Gel Recovery Kit (Axygen, USA).

2.3.3. Illumina PE300 Sequencing and Data Analysis. The amplicons were sequenced using the Illumina MiSeq PE300 platform (Illumina, USA), Miseq Reagent kit v3 (600-cycle) (Illumina, USA), by Shanghai Biozeron Biotech Co., Ltd (Shanghai, China).

The Illumina PE300 sequencing data first included the effective sequences of all samples. Based on the overlap between paired-end (PE) reads, the paired reads were spliced into a sequence. Finally, according to the barcode and primer sequences, the high-quality sequences of each sample were obtained by splicing, and the sequence direction was corrected according to the forward and reverse barcodes, and the direction of the primer was used in the process. Chimeras were removed, and finally, a valid sequence file was obtained. Statistical analysis of the bioinformation of each sample's OTUs was performed using Usearch (version10: http://drive5.com/uparse/) software at a similarity level of $97 \%$ by categorization.

To obtain the species classification corresponding to each OTU, the RDP classifier [22] was used to classify the $97 \%$ similarity level of representative OTU sequences, and the community composition of each sample was calculated at 
TABLE 1: Summary of HTS of the ITS1 amplicon for E. sinensis.

\begin{tabular}{lccc}
\hline & ES1 & ES2 & ES3 \\
\hline Sequence & 131,405 & 143,763 & 119,127 \\
Average length (bp) & 225 & 237 & 218 \\
Bases (bp) & $29,564,446$ & $34,174,249$ & $26,001,838$ \\
No. of OTUs & & 286 & 23 \\
No. of genera & 92 & 140 & 11 \\
\hline
\end{tabular}

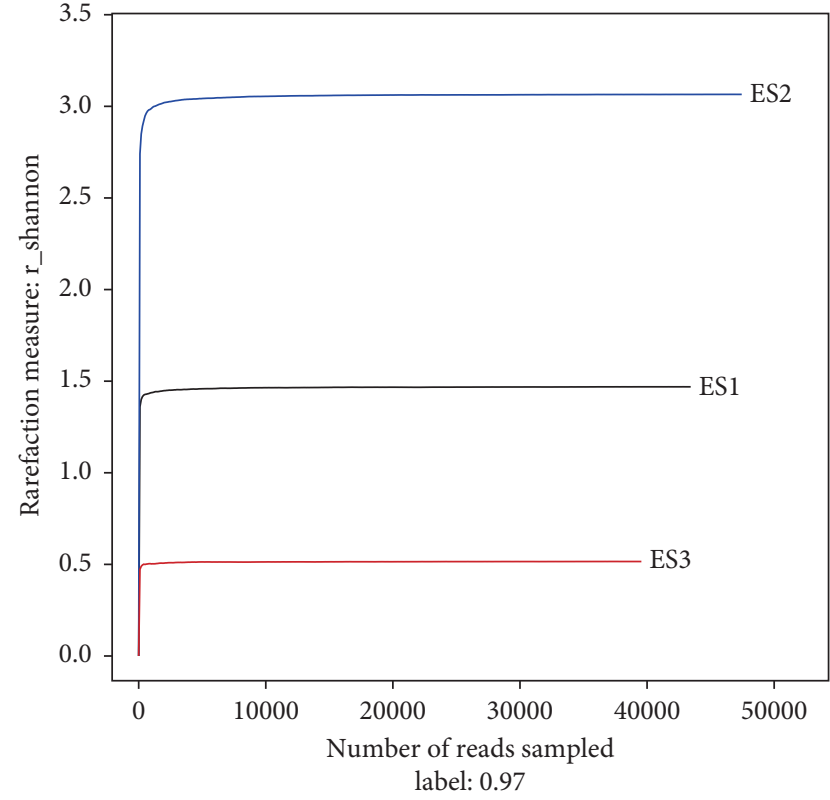

(a)

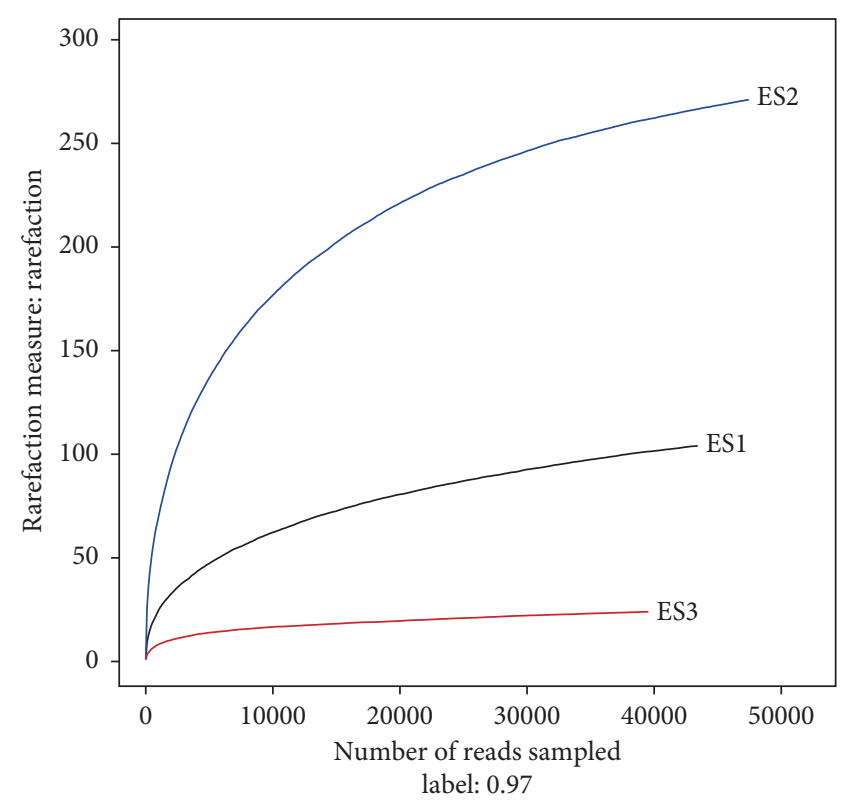

(b)

FIGURE 1: Evaluation of HTS data based on the ITS1 amplicon. Shannon-Wiener curves show that the fungal diversity levels off with deeper sequencing in all three E. sinensis samples (a). Rarefaction curves show that the abundance of fungal species increased with the number of reads $(b)$.

each classification level (family, genus, and species). Alpha diversity values of the Shannon index [23] (http://www. mothur.org/wiki/Shannon) and the Simpson diversity index (http://www.mothur.org/wiki/Simpson)were calculated using mothur (version v.1.30.1 http://www.mothur.org/ wiki/Schloss_SOP\#Alpha_diversity) software [24]. The richness of the species in the samples with different sequencing data was compared by rarefaction curve [25].

\section{Results and Discussion}

The culture-based dilution plating method was used for the separation of fungi in the three E. sinensis samples (ES1, ES2, and ES3). A total of 32 fungal clones was acquired from all samples. We extracted genomic DNA from each of the 32 clones and sequenced ITS and CaM PCR products using Sanger sequencing. By comparing ITS and CaM sequences against the NCBI database with the BLAST tool, a total of six fungal species were identified, including Aspergillus candidus, A. pseudoglaucus, A. ruber, A. tubingensis, Penicillium chrysogenum, and Hyphopichia burtonii.
For HTS analysis, community DNA was extracted from each of the three E. sinensis samples in triplicate. A total of nine DNA samples were analysed using Illumina MiSeq PE300 based on the amplicon sequencing of the fungal ITS1 region. The average length of the amplicon is about $227 \mathrm{bp}$. A total of 394,295 sequences were obtained (Table 1). After PE alignments, quality filtering, and deletion of chimeras and singletons, more than 100,000 ITS1 sequences from each sample were recovered. Shannon rarefaction analysis shows that the sequence data for each sample are saturated (Figure 1). A total of 286 OTUs were found in E. sinensis. The taxonomically and functionally annotated sequence database NCBI nucleotide $(\mathrm{Nt})$ was used to identify 140 fungal genera. In samples ES1 and ES3, Aspergillus was the majority fungal genus, representing $96.9 \%$ and $99.3 \%$ of genera, respectively. However, species-level taxonomy showed no $A$. ruber or $A$. tubingensis, which were found using the culture-based diluting plate method. In sample ES1, 25\% of sequences were annotated to Aspergillus section Aspergillus (formerly the genus Eurotium) but cannot be identified to species (Figure 2). 


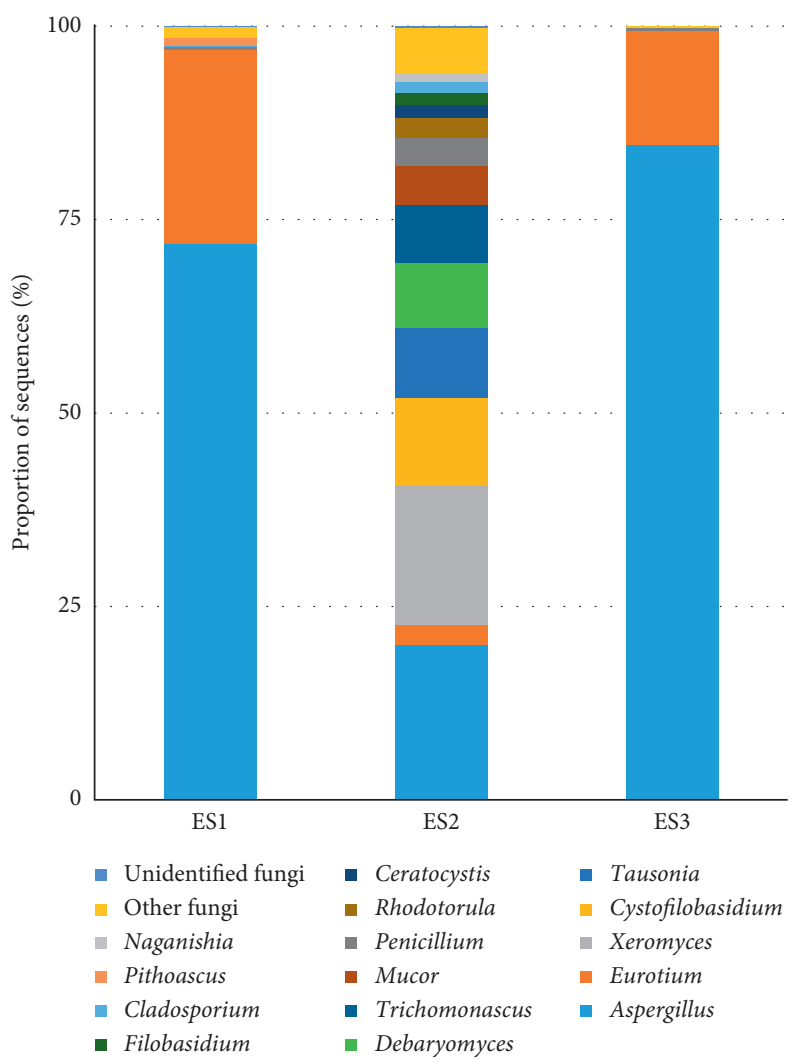

FIgURE 2: Genus-level percentage of fungal composition based on HTS of the ITS1 amplicon for all three E. sinensis samples.

Using the HTS platform, a portion of the CaM gene sequence was used to identify undefined Aspergillus sp. in $E$. sinensis samples. In Aspergillus sp., the CaM PCR product length obtained with previously described primers was about $600 \mathrm{bp}$ [16], which is too long for HTS. The three E. sinensis samples were sequenced in triplicate using the two primers CMD5 and CaMr1. The average length of the amplicon was $249 \mathrm{bp}$. A total of $1,026,856$ sequences were obtained (Table 2). The Shannon rarefaction analysis shows that the sequencing data for each sample are saturated (Figure 3). A total of 193 OTUs were found. When the taxonomically and functionally annotated sequence database $\mathrm{Nt}$ was used, only 13 fungal genera were found. The number of fungal genera identified by sequencing $\mathrm{CaM}$ was far fewer than the number of fungal genera identified by sequencing ITS1. This is because CaM sequences are abundant in Aspergillus sp., while being rarer in other fungi. Nineteen Aspergillus species were annotated based on the HTS of CaM (Figure 4). All four Aspergillus species (A. candidus, A. pseudoglaucus, A. ruber, and A. tubingensis) found using the culture-based diluting plate method were also found by HTS based on the CaM amplicon. In addition, another 15 Aspergillus species were found in $E$. sinensis samples, including potential toxigenic fungi $A$. flavus.

ITS is the primary fungal barcode marker used to identify individual strains and analyse fungal diversity in environmental samples [26]. In addition to the universal ITS1 and ITS4 primers, most primers targeting the ITS region were published in the 1990s $[8,17]$. Other ITS primers targeting ITS1 or ITS2 region were more recently
TABLe 2: Summary of the HTS of CaM amplicon for E. sinensis.

\begin{tabular}{lccc}
\hline & ES1 & ES2 & ES3 \\
\hline Sequence & 386,069 & 423,179 & 217,608 \\
Average length (bp) & 247 & 250 & 250 \\
Bases (bp) & $95,284,351$ & $105,669,488$ & $54,406,592$ \\
No. of OTUs & 169 & 193 & \\
No. of genera & & 72 & 60 \\
& 10 & 13 & 2 \\
\hline
\end{tabular}

designed to make better use of HTS [18, 27-29]. In this study, we used the ITS1-F and ITS2 primers, which generate PCR products no more than $300 \mathrm{bp}$ in size and are suitable for HTS. The amplicon is efficient at distinguishing fungal genera in E. sinensis. However, some studies have indicated that the ITS sequence is highly conserved in the Aspergillus section Aspergillus (formerly the genus Eurotium) and cannot be used to distinguish different species [10]. CaM is a $\mathrm{Ca}^{2+}$ sensor involved in a large number of biological processes. In a previous study, the CaM sequence was used to differentiate Aspergillus species. In order to identify the potential toxigenic Aspergillus species using HTS in E. sinensis, we designed a new CaM primer which could be used in HTS analysis. Thus, CaM HTS analysis can be used to identify Aspergillus species in community DNA samples, making up for ITS1 deficiencies.

In the ES1 sample, $10.9 \%$ sequences were annotated to A. flavus; however, it was not detected through the 


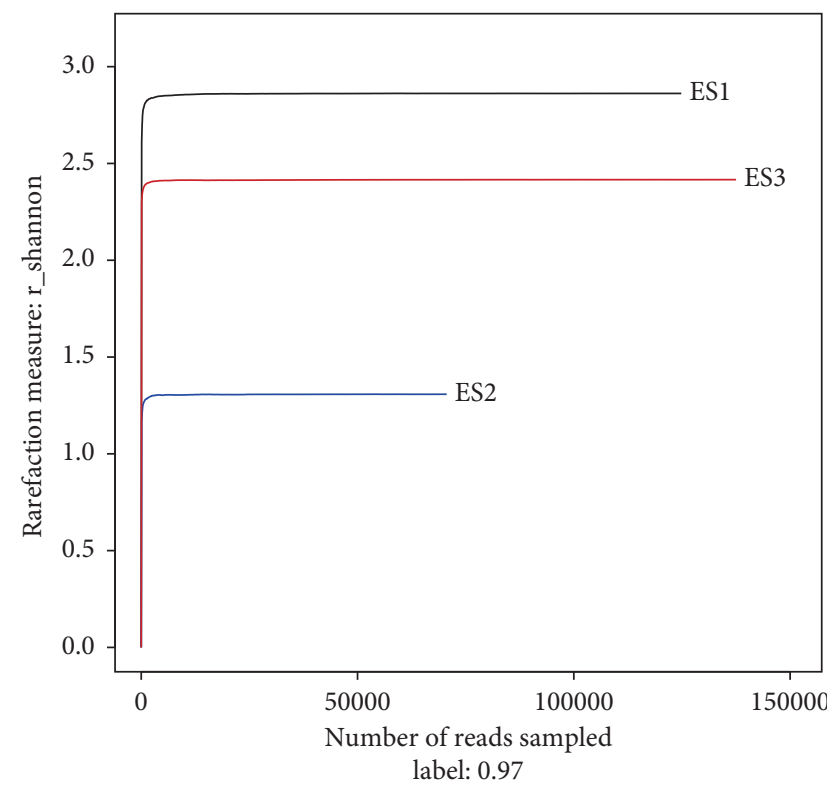

(a)

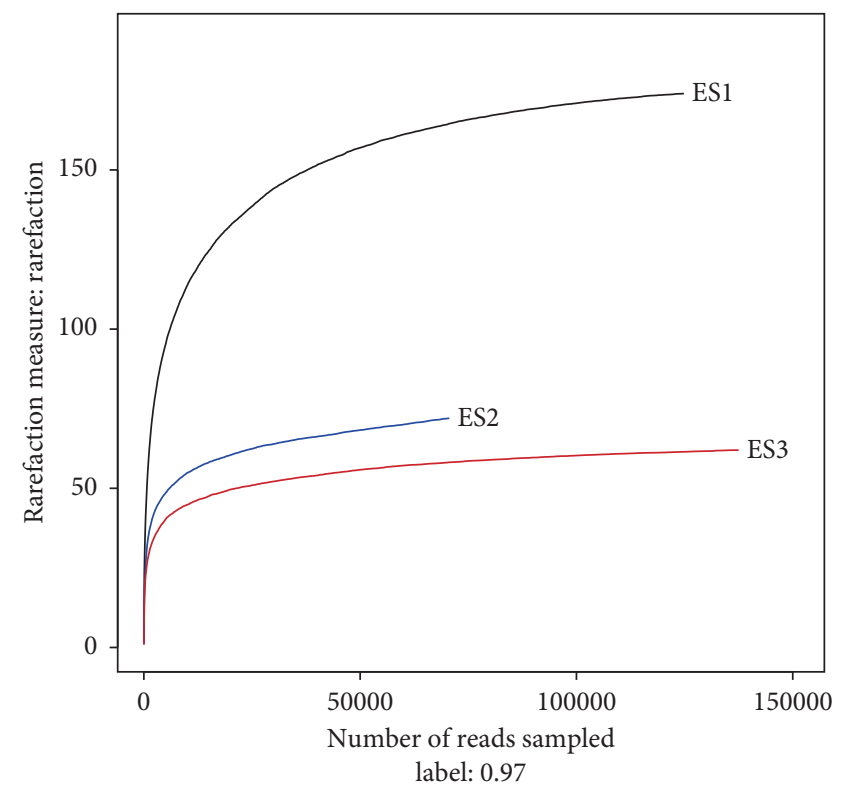

(b)

FIGURE 3: Evaluation of HTS data based on the CaM amplicon. Shannon-Wiener curves show that the fungal diversity levels off with deeper sequencing in all three E. sinensis samples (a). Rarefaction curves show that the abundance of fungal species increased with the number of reads (b).

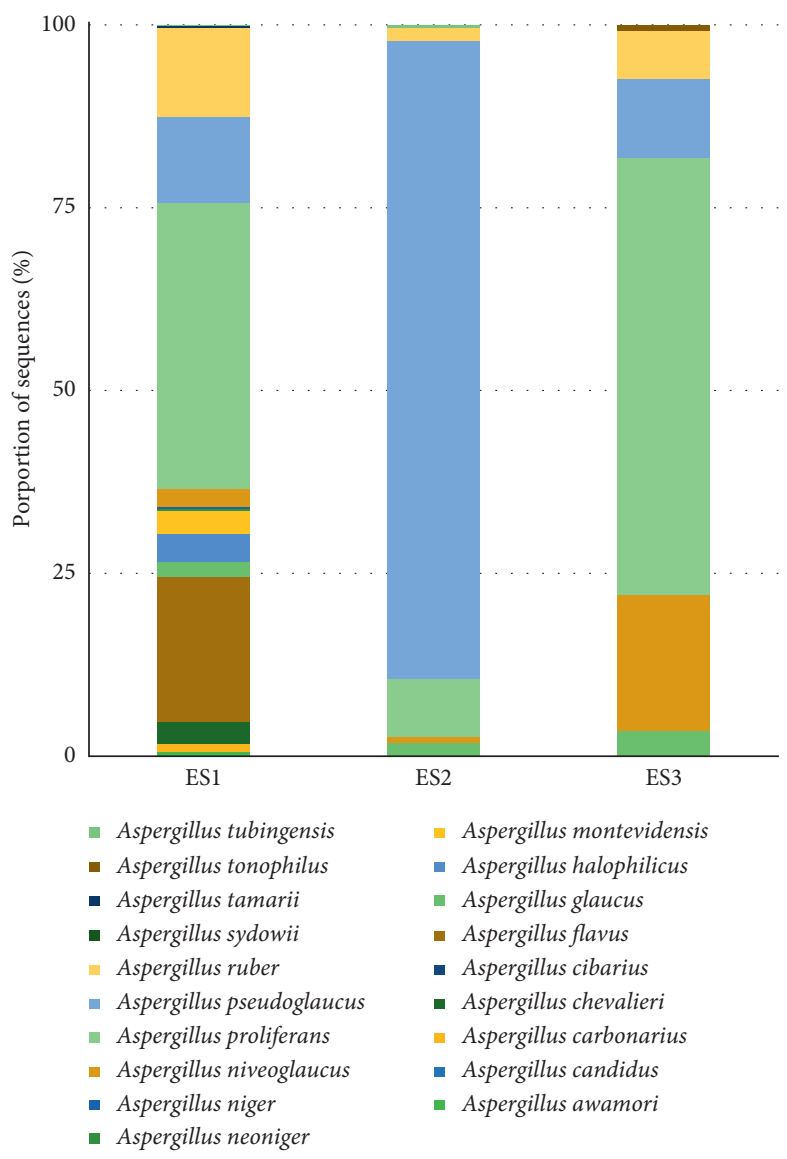

FIgUre 4: The percentage of Aspergillus species based on HTS of the CaM amplicon for all three E. sinensis samples. 
culture-based dilution plating method. It is known that a high proportion of fungi cannot currently be cultured under available artificial conditions [30]. Although we crushed the E. sinensis samples, fungi on residual feed in the gut are difficult to isolate. The HTS can much more effectively identify the internal fungi.

In the ES3 sample, there were only two sequences annotated to A. flavus through HTS, while the culture-based dilution plating method could not separate A. flavus from the sample. The trace amount of toxigenic A. flavus in $E$. sinensis is difficult to isolate through the culture-based dilution plating method. Under improper storage conditions, the potential toxigenic fungi may bloom in E. sinensis. In addition, HTS technology only requires several hours, whereas the culture-based dilution plating method takes nearly half a month. Overall, HTS analysis of the fungal microbiome is better than the culture-based method for early detection of potential toxigenic fungi in E. sinensis. Our results show that HTS is highly effective for identifying toxigenic fungi in the animal medicines $E$. sinensis.

It is important to study the Aspergillus sp. in the foods and the medicines in order to stay away from mycotoxins. The next step in the development of HTS as a screen for mycotoxins may be detection of the aflatoxin genes themselves. The biosynthetic pathway of aflatoxin has been completely elucidated, and the expression level of two genes, aflS and aflR, regulates the transcription of the structural genes in the aflatoxin cluster [31,32]. The expression level of the two genes may reflect the level of aflatoxin production. Next, we may detect the expression of aflS or aflR based on HTS to predict whether there is potential toxigenic fungi in TCM.

\section{Conclusions}

We used the culture-based dilution plating method and HTS to study the fungal composition of three E. sinensis samples. The number of contaminant fungal genera and species identified by HTS was clearly much higher than that obtained by using the culture-based dilution plating method. Based on HTS of the ITS1 amplicon, 286 OTUs annotated to 140 fungal genera were found. Aspergillus was the dominant fungal genus in all three E. sinensis samples. A total of 19 Aspergillus species were found based on HTS of the CaM amplicon. Among them, only four Aspergillus species were found based on the culture-based dilution plating method. There were some sequences of A. flavus found in ES1 and ES3 through HTS. Combined HTS of ITS1 and CaM is an effective early warning method for monitoring potential toxigenic fungi and mycotoxins in TCM.

\section{Data Availability}

Raw sequences are available in the Sequence Read Archive of the NCBI under the accession number PRJNA598990.

\section{Conflicts of Interest}

The authors declare that there are no conflicts of interest.

\section{Acknowledgments}

This work was supported by the Open Project of State Key Laboratory of Plateau Ecology and Agriculture, Qinghai University (2017-ZZ-15), CAS "Light of West China” Program, and CAMS Innovation Fund for Medical Sciences (CIFMS, grant no. 2017-I2M-1-013).

\section{References}

[1] N. Zhang, Y. Zhao, Y. Shi, R. Chen, X. Fu, and Y. Zhao, "Polypeptides extracted from Eupolyphaga sinensis walker via enzymic digestion alleviate UV radiation-induced skin photoaging," Biomedicine \& Pharmacotherapy, vol. 112, Article ID 108636, 2019.

[2] W. Zhang, Y. H. Zou, and L. G. Zhu, "Content determination of aflatoxins in Eupolyphaga by HPLC," China Pharmacy, vol. 26, no. 30, pp. 4269-4271, 2015.

[3] L. N. Liu, Y. L. Li, H. Y. Jin, and S. C. Ma, "Determination of aflatoxins in animal medicines by immunoaffinity column and HPLC-FLD with photochemical derivatization fluorescence detection," Chinese Traditional and Herbs Drugs, vol. 48, no. 6, pp. 1220-1224, 2017.

[4] P. H. Yu, L. Zhang, J. A. Qin, J. H. Wang, S. H. Yang, and M. H. Yang, "Simultaneous determination of aflatoxin B1, B2, G1, G2, M1, M2 in Eupolyphaga Steleophaga by immunoaffinity column clean-up and HPLC-FLD with postcolumn photochemical derivatization," China Journal of Chinese Materia Medica, vol. 44, no. 23, pp. 5083-5087, 2019.

[5] N. W. Turner, H. Bramhmbhatt, and M. Szabo-Vezse, "Analytical methods for determination of mycotoxins: an update (2009-2014)," Analytica Chimica Acta, vol. 27, pp. 3232-3235, 2015.

[6] G. Y. Ying, X. Zhao, and J. L. Wang, "Application and prospect of "medicine pair" technology in anti-mildew maintenance of Chinese medicinal materials," Chinese Journal of Traditional Chinese Medicine, vol. 41, pp. 2768-2773, 2016.

[7] G. A. Payne and M. P. Brown, "Genetics and physiology of aflatoxin biosynthesis," Annual Review of Phytopathology, vol. 36, no. 1, pp. 329-362, 1998.

[8] T. J. White, T. Bruns, S. Lee, and J. Taylor, "Amplification and direct sequencing of fungal ribosomal RNA genes for phylogenetics," in PCR Protocols a Guide to Methods and Applications, M. A. Innis, D. H. Gelfand, J. J. Sninski, and T. J. White, Eds., Academic Press, Inc, San Diego, CA, USA, 1990.

[9] C. L. Schoch, K. A. Seifert, S. Huhndorf et al., "Nuclear ribosomal internal transcribed spacer (ITS) region as a universal DNA barcode marker for fungi," Proceedings of the National Academy of Sciences of the United States of America, vol. 109, pp. 6241-6246, 2012.

[10] A. J. Chen, V. Hubka, J. C. Frisvad et al., "Polyphasic taxonomy of Aspergillus section Aspergillus (formerly Eurotium), and its occurrence in indoor environments and food," Studies in Mycology, vol. 88, pp. 37-135, 2017.

[11] R. A. Samson, C. M. Visagie, J. Houbraken et al., "Phylogeny, identification and nomenclature of the genus Aspergillus," Studies in Mycology, vol. 78, pp. 141-173, 2014.

[12] D. B. Müller, C. Vogel, Y. Bai, and J. A. Vorholt, "The plant microbiota: systems-level insights and perspectives," Annual Review of Genetics, vol. 50, no. 1, pp. 211-234, 2016.

[13] A. C. Huang, T. Jiang, Y. X. Liu et al., "A specialized metabolic network selectively modulates Arabidopsis root microbiota," Science, vol. 364, p. 6440, 2019. 
[14] W.-J. Lee and K. Hase, "Gut microbiota-generated metabolites in animal health and disease," Nature Chemical Biology, vol. 10, no. 6, pp. 416-424, 2014.

[15] N. Zmora, J. Suez, and E. Elinav, "You are what you eat: diet, health and the gut microbiota," Nature Reviews Gastroenterology \& Hepatology, vol. 16, no. 1, pp. 35-56, 2019.

[16] S.-B. Hong, S.-J. Go, H.-D. Shin, J. C. Frisvad, and R. A. Samson, "Polyphasic taxonomy of Aspergillus fumigatus and related species," Mycologia, vol. 97, no. 6, pp. 1316-1329, 2005.

[17] M. Gardes and T. D. Bruns, "ITS primers with enhanced specificity for basidiomycetes - application to the identification of mycorrhizae and rusts," Molecular Ecology, vol. 2, no. 2, pp. 113-118, 1993.

[18] K. J. Martin and P. T. Rygiewicz, "Fungal-specific primers developed for analysis of the ITS region of environmental DNA extracts," BMC Microbiology, vol. 5, no. 1, p. 28, 2005.

[19] M. Buée, M. Reich, C. Murat et al., "454 Pyrosequencing analyses of forest soils reveal an unexpectedly high fungal diversity," New Phytologist, vol. 184, no. 2, pp. 449-456, 2009.

[20] M. A. Ghannoum, R. J. Jurevic, P. K. Mukherjee et al., "Characterization of the oral fungal microbiome (Mycobiome) in healthy individuals," PLoS Pathogens, vol. 6, no. 1, Article ID e1000713, 2010.

[21] K. L. McGuire, S. G. Payne, M. I. Palmer et al., "Digging the New York city skyline: soil fungal communities in green roofs and city parks," PLoS One, vol. 8, no. 3, Article ID e58020, 2013.

[22] Q. Wang, G. M. Garrity, J. M. Tiedje, and J. R. Cole, "Naive Bayesian classifier for rapid assignment of rRNA sequences into the new bacterial taxonomy," Applied and Environmental Microbiology, vol. 73, no. 16, pp. 5261-5267, 2007.

[23] W. Yu and H. F. Sheng, "Comparison of the levels of bacterial diversity in freshwater, intertidal wetland, and marine sediments by using millions of Illumina tags," Applied and Environmental Microbiology, vol. 78, no. 23, pp. 8264-8271, 2012.

[24] P. D. Schloss, D. Gevers, and S. L. Westcott, "Reducing the effects of PCR amplification and sequencing artifacts on $16 \mathrm{~S}$ rRNA-based studies," PLoS One, vol. 6, no. 12, Article ID e27310, 2011.

[25] P. D. Schloss, S. L. Westcott, T. Ryabin et al., "Introducing mothur: open-source, platform-independent, communitysupported software for describing and comparing microbial communities," Applied and Environmental Microbiology, vol. 75, no. 23, pp. 7537-7541, 2009.

[26] H. E. O’Brien, J. L. Parrent, J. A. Jackson, J.-M. Moncalvo, and R. Vilgalys, "Fungal community analysis by large-scale sequencing of environmental samples," Applied and Environmental Microbiology, vol. 71, no. 9, pp. 5544-5550, 2005.

[27] E. Bellemain, T. Carlsen, C. Brochmann, E. Coissac, P. Taberlet, and H. Kauserud, "ITS as an environmental DNA barcode for fungi: an in silico approach reveals potential PCR biases," BMC Microbiology, vol. 10, no. 1, p. 189, 2010.

[28] K. Ihrmark, I. T. M. Bödeker, K. Cruz-Martinez et al., "New primers to amplify the fungal ITS2 region - evaluation by 454sequencing of artificial and natural communities," FEMS Microbiology Ecology, vol. 82, no. 3, pp. 666-677, 2012.

[29] M. Op De Beeck, B. Lievens, P. Busschaert, S. Declerck, J. Vangronsveld, and J. V. Colpaert, "Comparison and validation of some ITS primer pairs useful for fungal metabarcoding studies," PLoS One, vol. 9, no. 6, p. e97629, 2014.
[30] J. L. Kirk, L. A. Beaudette, M. Hart et al., "Methods of studying soil microbial diversity," Journal of Microbiological Methods, vol. 58, no. 2, pp. 169-188, 2004.

[31] J. Yu, P.-K. Chang, K. C. Ehrlich et al., "Clustered pathway genes in aflatoxin biosynthesis," Applied and Environmental Microbiology, vol. 70, no. 3, pp. 1253-1262, 2004.

[32] P. Belén, R. Mar, D. Josué, J. A. María, and R. Alicia, "Gene expression as a good indicator of aflatoxin contamination in dry-cured ham," Food Microbiology, vol. 67, pp. 31-40, 2017. 